\title{
Johann Friedrich Mayer - der „Gypsapostel“ als Pfarrer und Prediger in Kupferzell
}

\author{
von Dietrich BLeher
}

Wer durch die Hohenloher Dörfer und Weiler kommt, freut sich immer wieder über die markanten, prächtigen Bauernhäuser - ein mit Steinen gemauertes Erdgeschoss, der Wirtschaftsbereich; der erste Stock in Fachwerk, oft mit schön beschnitzten Eckbalken als Wohnbereich, das Gebäude in stets gleicher Gliederung: Hauseingang in der Mitte, links und rechts je ein Stalleingang für Zugbzw. Nutzvieh. Ein typisches „Pfarrer-Mayer-Haus“, wird man auf Nachfrage immer wieder hören; ein Haus, wie es sich Pfarrer Johann Friedrich Mayer (1719-1798) als praktisch, wirtschaftlich, preiswert und schön für seine Landleute empfohlen hatte. In diesen Häusern ist sein segensreiches Wirken noch heute im wahrsten Sinne des Wortes sichtbar. Darüber hinaus weiß hier noch fast - jeder, dass der langjährige Ortspfarrer mit seinem reichen naturwissenschaftlichen Wissen Entwicklungsimpulse gegeben hat, die in Hohenlohe noch heute spürbar sind: Gyps-Düngung, verbesserte Drei-Felder-Wirtschaft, ganzjährige Stallhaltung des Viehs, Ochsenmast und -handel. Hohenlohe wurde so zum reichen Bauernland.

Von all dem ist im Hohenloher Freilandmuseum in Wackershofen manches sichtbar ausgestellt und beschrieben. Weniger sichtbar und kaum beschrieben ist dagegen, welche Rolle seine naturwissenschaftlichen, praktischen Erkenntnisse in seiner Verkündigung in Kupferzell (1745-1798) gespielt haben. Hat Mayer Naturwissenschaft verkündigt? Sind seine Einsichten biblisch theologisch begründet und verankert? Wie gebrauchte Mayer biblische Texte? Legte er sie aus oder waren sie bloß schmückendes Beiwerk seiner Naturlehre? Sprach er die „Landleute“ direkt an oder doch die ganze Gemeinde?

\section{Das Predigtbuch}

Solchen und ähnlichen Fragen möchte ich im Folgenden nachgehen, indem ich über seine Predigten berichte. Johann Friedrich Mayer war ein fleißiger Autor. Von 37 Veröffentlichungen von 1768 an sind 35 naturwissenschaftlich-praktischen Inhalts, also nur zwei theologisch, geistlich: ein Buch für den kirchlichen Unterricht der Jugend (1771) und ein Predigtbuch für die häusliche Andacht und Erbauung der Gemeinde. Das Buch über den kirchlichen Unterricht war mir zur 
Zeit der Abfassung meines Textes noch nicht zugänglich, liegt aber inzwischen in einem Digitalisat der Universitätsbibliothek Tübingen vor. Das Predigtbuch ${ }^{1}$ wurde erst zwei Jahre nach seinem Tod im Jahre 1800 von seinem Sohn herausgegeben unter dem Titel: Predigtbuch für christliche Bürger und Landleute hinsichtlich auf ächte Christus-Religion, wahre Lebensweisheit und kluge Haushaltung zur häuslichen Andacht und Vorlesung auf alle Sonn= und =Fest-Tage des ganzen Jahres nach den Evangelien [...], von Johann Friederich Mayer [...], Heilbronn und Rothenburg 1800. Der Band umfasst XXVIII und 780 Seiten.

Ziel und Zweck der Predigten ist es, dem christlichen Bürger und Landmann ächte, christliche Religion zu vermitteln, die die wahre Lebensweisheit ist, eine Lebensweisheit, durch die man mit wenigem vergnügt ist und mit vielem klug umzugehen und hauszuhalten wei $\beta .^{2}$ So hat der Herausgeber seinen Vater richtig verstanden. Das Inhaltsverzeichnis ist denn auch ein Katalog der Lebensweisheiten, durch deren Befolgung der Bürger und Landmann hier schon den Anfang der Seligkeiten der Ewigkeit genießt.

Ein paar Beispiele für solche Lebensweisheiten:

1. Advent: Wer seinem Beruf auf seine Wohlfahrt genug tun will, der muss sich auf ihn vorbereiten.

Weihnachten: Dass der Christ verbunden sey, auch einer heidnischen Obrigkeit, die Gewalt über ihn hat, zu gehorchen und sich in keinem Fall wider sie zu empören.

Sonntag nach Weihnachten: was uns vom Segen, um Vorteile aus ihm zu ziehen, zu wissen notwendig ist.

Epiphanias: dass es dem Christen Pflicht sei, alles zu wagen, um sein volles Glück zu machen. ${ }^{3}$

Die Lebensweisheiten berühren alle Lebensbereiche: Erziehung der Kinder Glück der Ehen - Herr und Knecht - Verhalten bei Naturkatastrophen - Umgang mit dem Bösen - Arbeit und Lohn - gute Vorsätze - Verlockungen durch Außergewöhnliches - Glück und Unglück - wie segnet Gott - sich Segen bewahren, gegen Verderben schützen - Reichtum und Armut - Einnahmen und Ausgaben - Neid; Gesundheit und Krankheit - Dankbarkeit - Pflichten der Lebenden gegenüber Sterbenden und Toten. usw.

1 Im Hohenlohe-Zentralarchiv Neuenstein war das Buch nicht vorhanden. In der Landesbibliothek in Stuttgart gibt es ein Exemplar - Ausleihe schwierig. Im Stahlschrank im Kupferzeller Pfarramt - wie es sich gehört - ein Exemplar - allerdings ohne das ,wohlgetroffene Bildnis des seligen Verfassers" - irgendjemand hat es herausgeschnitten und geklaut! Unter diesen Gegebenheiten konnte ich mich an die Lektüre machen. Erst nach Fertigstellung meines Aufsatzes erhielt ich - dank einer Begegnung mit Archivdirektor i. R. G. Franz, Trier, den Hinweis auf eine inzwischen bessere Quellenlage. So wurde „Die Lehre der evangelischen Kirche zum Unterricht für die Jugend“ 2016 von der Universitätsbibliothek Tübingen digitalisiert und die Universitätsbibliothek Erlangen-Nürnberg stellt jetzt das Predigtbuch - selbstverständlich mit Porträt - online zur Verfügung.

2 Vorrede S. IX. Wenn in den nachfolgenden Fußnoten nichts anderes angegeben ist, beziehen sich die dort genannten Seitenzahlen immer auf Mayers Predigtsammlung.

3 1. Advent: S. 1; Weihnachten: S. 45; Sonntag nach Weihnachten: S. 57; Epiphanias: S. 90. 
Auch hinter deutlicher biblisch-theologisch klingenden Stichworten stehen lebenspraktische Ratschläge:

- Gebet (Sonntag Rogate)

- Glaube (Himmelfahrt)

- Gottes Größe in Rat und Tat (Pfingsten)

- Glaube, Unglaube, Aberglaube: Was dem Landmann wirklich nützt und scha$\operatorname{det}^{4}$

Die Sonntagsthemen sind im Inhaltsverzeichnis ohne einen dazugehörigen Bibeltext aufgeführt. Umso spannender die Frage: Welchen Evangeliumstext wählte Mayer zu welchem Thema? Für mich war nur ein einziges Mal schon im Inhaltsverzeichnis klar, welcher Text an diesem Sonntag „dran sein“ musste: Sexagesimä - die Verschiedenheit des Ackers. Das kann nur die Geschichte vom vierfachen Ackerfeld sein (Lukas 8, 4-15).

Aber wie kommt Mayer sonst von seinem Thema zum Text oder eher vom biblischen Text gerade zu diesem Thema? Ein Blick in die Predigten zeigt, sie sind durchweg nach dem gleichen Schema verfasst:

a) Evangeliumstext -

b) pädagogischer Gedanke - c) Entfaltung des Themas in Verbindung mit dem Text $-\mathrm{d}$ ) Vorschlag, Rat, Lehre für die Landleute.

Als Beispiel wähle ich den Sonntag Esto mihi. ${ }^{5}$

a) Predigttext ist Lukas 18, 31-43, die Geschichte von dem Blinden in Jericho, der den vorübergehenden Jesus doch wahrnimmt und um sein Erbarmen bittet. Gegen die ihn wegdrängende Menge ruft er noch einmal nach Jesus, der sich dann doch ihm zuwendet.

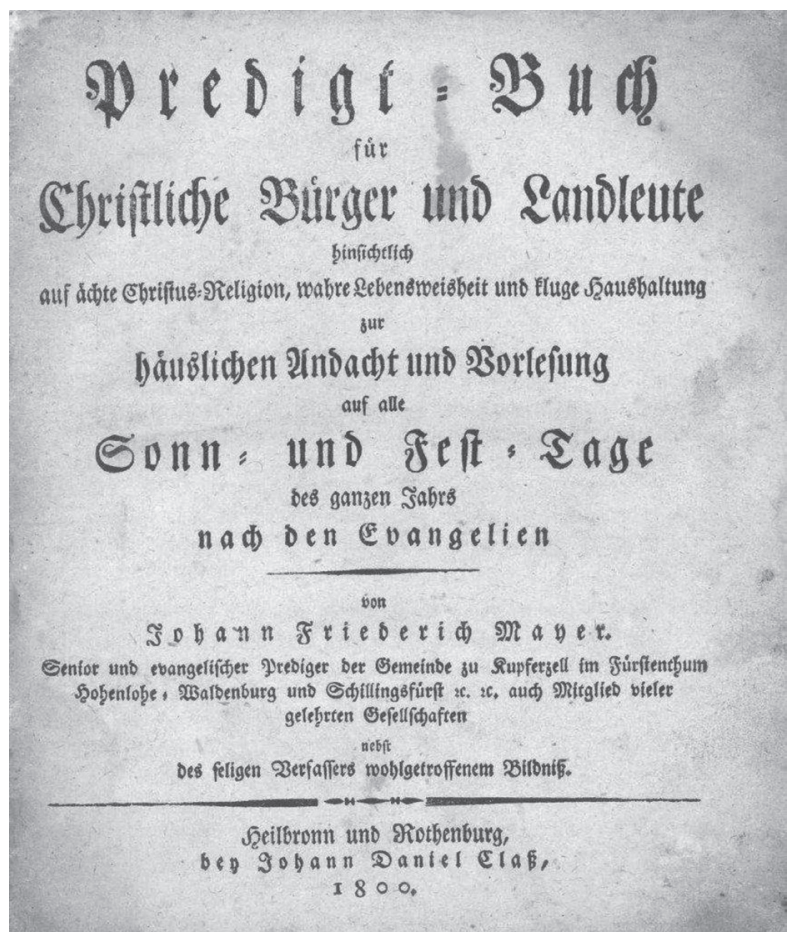

Titelblatt von Johann Friedrich Mayers Predigtbuch 
Jetzt kann er seine Bitte um Heilung aussprechen. Und Jesus macht ihn sehend. „Dein Glaube hat Dir geholfen.“

b) Pädagogischer Gedanke: Mayer beginnt häufig mit Worten aus den Sprüchen oder aus Sirach, wie hier $(2,14)$ : ,Weh dem, der an Gott verzagt und nicht festhält und dem Gottlosen, der hin und her wankt.“ Ein Grundübel des Menschen ist die Wankelmütigkeit. Beispiele dafür sind: Kain, Jonas, Saul. Sie wurden von Gott gestraft. Anders Johannes der Täufer.

So geht es bis heute jedem, der an seinem Vorsatz nicht festhält, zumal den Landleuten, die sich über ihren Geschäften nur immer hin besinnen, rathen, anlaufen wieder ablassen, zurücktreten, sich immer Mühe machen und so niemalen oder sehr selten zum Zweck kommen. Darinnen liegt nun ihr Unglück. Ihnen wäre wohl ein besseres Schicksal zu wünschen und darum geschieht auch heute nach Anleitung des Ev. ein Vorschlag, der dieser ist und ihnen als Anforderung an sie vorgetragen werden soll. ${ }^{6}$

Es folgt das im Inhaltsverzeichnis genannte Thema: Einen einmal weislich gefassten Entschluß muß man schlechtweg nicht wieder aufgeben.

c) Mayer nennt die zerrinnende, begrenzte Zeit des Menschen, die es auszukaufen gilt, wenn man ein angestrebtes, gutes Ziel - Wohlfahrt und Beglückung erreichen will. Als Christ verfolgt man natürlich keine törichten Ziele. Wie der Blinde überlegt der Christ, wie er am besten zum Ziel kommt und setzt demgemäß seine Mittel ein. Glaube erweckt Mut und Standhaftigkeit und überwindet Hindernisse.

So war und ist es ja auch bei Jesus selber auf dem Weg nach Jerusalem. Er lässt sich nicht aufhalten, überwindet Spott und Hohn, Gericht, Tod und Hölle. Der Sieg seines Entschlusses: die Erlösung, die Beglückung des Menschen. Das ist Reiz für uns alle, einen weislich gefaßten, guten Entschluß nie wieder aufzugeben bis er glücklich vollendet ist. ${ }^{7}$

d) Diese Auslegung wendet Mayer nun auf die Landleute an. ${ }^{8}$ Von ihrer klug geplanten, zielstrebigen Arbeit hängen Wohl und Wehe der ganzen Gesellschaft ab. Wo sie sich selbst hindern - durch falsche Arbeitstechnik, Unkenntnis, Unbelehrbarkeit, Faulheit etc. - müssen sie sich korrigieren, dazulernen und Einsicht, Fleiß verdoppeln. Wo andere sie abhalten, müssen diese überwunden werden. Der Landleute Arbeiten erfordern wahrlich mehr Mut und Nachdenken, als viele glauben zu wissen. ${ }^{9}$

Mayer schließt mit zwei Liedversen, die vom Vertrauen geprägt sind, dass das menschlich - bäuerliche Tun - von Gott wohlgefälligen Entschlüssen und Beständigkeit getragen - am Ende zu einem guten Ergebnis, zur wahren Beglückung des Menschen führt. ${ }^{10}$

\footnotetext{
6 Ebd., S. 202.

7 Ebd., S. 209.

8 Ebd., S. 210.

9 Ebd., S. 212.

10 Ebd., S. 213, Lied.
} 
Was hier in diesem Beispiel noch klar als Textauslegung erscheint - auf den Gedanken der Konsequenz konzentriert - verwischt sich in vielen anderen Predigten, weil zu viele - isolierte - Bibelworte eingestreut und immer wieder die gleichen Verhaltensweisen mit den entsprechenden Ratschlägen gebracht werden. Der Zusammenhang mit dem Evangeliumstext ist dann oft recht dünn. Dem aufmerksamen Leser kommt schon nach wenigen Predigten vieles bekannt vor. Mayers Predigten sind nach Inhalt und Methode Produkt einer von der Aufklärung und vom Rationalismus geprägten Zeit. Sie geht davon aus, dass diese Welt als Schöpfung eines gütigen Gottes alles enthält, was der Mensch zu seiner Wohlfahrt und Glückseligkeit braucht. Das wissen und feiern Christen am Neujahrstag - dem Fest der Namengebung Jesu. ${ }^{11}$ Jesus heißt und ist: der Seligmacher. Der Mensch ist dazu bestimmt, durch Jesus Seligkeiten ohne Ende zu empfangen. ${ }^{12}$ nicht erst in der Ewigkeit, sondern schon hier und jetzt. Gott hat die Erde mit Gutem gefüllt. Der Mensch ist von Gott so ausgestattet mit Hand und Fuß, Kräften des Leibes und der Seele, dass er diese Schätze heben und genießen kann. Durch Gottes Wort ist alles gesagt, was der Mensch braucht. Er muss nur seine Vernunft sprechen lassen und die Belehrung Jesu beachten.

Sei fleißig, dein Glück aus den Tiefen zu graben und scheue die Arbeit niemals, nur der Lässige ти $\beta$ zinsen (Sprüche 12, 24).

Der Mensch braucht sich aber nicht zu überfordern. Gott hat jedem das Seine zugemessen, jedem seinen Platz, sein Gewerb, seine Arbeit. So auch dem Landmann. Es ist wohl kein Beruf, der so viel Gutes und Glänzendes für sich hat und aufweisen kann als der des Landmanns. ${ }^{13}$

Letztlich zielen die Predigten immer wieder darauf, das weise Verhalten des Christen in unterschiedlichsten Lebenssituationen herauszufinden und einzuüben. Dem Bürger und Landmann dabei zu helfen ist die Aufgabe des Pfarrers. Er ist der Lehrer seiner Gemeinde.

Ein gutes Beispiel für den Erfolg der Vernunftanstrengung ist der Blitzableiter. Mayer erwähnt ihn am 4. Sonntag nach Epiphanias in der Predigt über Matthäus 8, 23-27 - die Stillung des Sturms auf dem See Genezareth. Unter dem Thema Das weise Verhalten des Christen bei der sich wider sein Glück zu empören scheinenden Natur nennt er allerlei Naturgewalten, die den Menschen bedrohen können. Für Mayer könnte der Blitzableiter zum Zeitpunkt der Predigtabfassung - doch wohl schon Jahre vor der Herausgabe des Bandes - noch so neu gewesen sein, dass er seine Wirkung noch in einem Fragesatz ausdrückt. Könnte nicht das Einschlagen der Blitze, wie es die Erfahrung aus untrüglichen Gründen und Ursachen beweist, durch wohltätigen Gewitter-Ableiter verwehrt werden? ${ }^{14}$ 
Mayer jr. als Herausgeber macht an dieser Stelle eine ihm notwendig erscheinende Anmerkung und fügt die neueste Beschreibung der naturwissenschaftlichen Zusammenhänge an. Offensichtlich ist die Belehrung durch Pfarrer Mayer in seiner Gemeinde und der Umgebung nicht begeistert aufgenommen worden. Die Hausbesitzer waren skeptisch - ein eindrücklicher Beweis dafür, wie hartnäckig die vernünftige christliche Belehrung manchmal sein muss, um ein gutes Ziel zu erreichen.

Mayer lebte fast 50 Jahre im beschaulichen Amtsstädtchen der Herrschaft Hohenlohe-Waldenburg-Schillingsfürst. Da war die Welt - noch - in Ordnung, das Verhältnis Staat-Kirche - auch bei einer katholischen Herrschaft - klar geregelt, eingespielt und ungetrübt. So war der katholische Fürst im evangelischen Waldenburg selbstverständlich Patronatsherr und hatte in der evangelischen Stadtkirche seine Fürstenloge.

Umso aufregender waren die Ereignisse in der Welt draußen. Mayer hat von Kupferzell aus die Französische Revolution und den Aufstieg Napoleons miterlebt. Das wird auch die Herrschaft beschäftigt und manche Befürchtungen ausgelöst haben. Zur Zeit der Herausgabe des Predigtbandes 1800 waren die schlimmsten Ereignisse der Französischen Revolution schon Geschichte und Napoleons Aufstieg im Gange. Der Herausgeber bringt zwei Predigten, die sich ganz direkt dem Gegenüber von Herrschaft und Bürgern widmen:

Am Fest der Geburt Christi: daß der Christ verbunden sei, auch einer heidnischen Obrigkeit, die Gewalt über ihn hat, zu gehorchen und sich in keinem Falle wider sie zu erheben.

Evangeliumstext der Predigt ist die Weihnachtsgeschichte Lukas 2, 1-14. ${ }^{15}$

Im ganzen Predigtband kommen Juden nur im Zusammenhang mit Bibeltexten vor, nicht als Bürger oder Geschäftspartner. Über die Erörterung der Frage von Juden, ob man dem römischen Kaiser Steuern zahlen dürfe (Matthäus 22,17) und Jesu Antwort, kommt Mayer zu seinem Thema. Die Geburtsgeschichte ist ihm Beweis für das Verabscheuungswürdige des Aufruhrs, hat doch Jesus seine Geburt unter einem heidnischen Kaiser akzeptiert und die Ausführung seines Auftrags als Seligmacher der Menschen dennoch für möglich gehalten. Von Gott geschieht nichts und wird nichts zugelassen und bestimmt, womit er nicht weise und gute Absichten allezeit verbinde. ${ }^{16} \mathrm{Zu}$ Aufruhr besteht keinerlei Anlass, haben wir doch auch unter ihr (sc. der heidnischen Obrigkeit) noch Gelegenheit, unser Brod zu erwerben, unser Gewerbe zu treiben und Sicherheit wegen unserem Eigentum. Und selbst unter einer heidnischen Obrigkeit kann Gott gepriesen werden Ehre sei Gott in der Höhe und Frieden auf Erden. Gegen einen ungerechten Herrscher darf sich der Christ wehren - im Rahmen des Rechts, nicht durch Aufruhr. Mit gewalttätigem Aufruhr schadet sich der Bürger nur selbst. Er zerstört letztlich seine Wohlfahrt, denn das ganze Land wird ausgeplündert und 
zerstört. Darum sind die Landleute wohl klug, wenn sie dem Feldgeschrei des Aufruhrs nicht nachlaufen, sondern still bei ihrem friedlichen Herrn bleiben, Gott fürchten, den König, die Obrigkeit ehren.

Auch bei dieser Predigt findet sich eine der wenigen Anmerkungen des Herausgebers. Er weist darauf hin, dass oft nicht alles Gold ist, was glänzt. 1000 Ursachen machen den Großen der Erde die Pracht und den Glanz notwendig, so beschwerlich er ihnen auch ist. ${ }^{17}$

Eine eigenartige Argumentation!

Die zweite, auf die Obrigkeit bezogene Predigt wird am Palmsonntag präsentiert. Sie ist ein Lobpreis der Gerechtigkeit Kaiser Josephs II. und für den Leser als Reiz zu seiner Verehrung und Hochschätzung gedacht. Mit dem für Palmsonntag vorgesehenen Evangeliumstext hatte Mayer schon die Leichenrede auf Joseph II. gehalten. Deshalb greift er in dieser Predigt auf Psalm 112, 6 f. zurück. ${ }^{18}$

Mayers Begründung für ein Abweichen vom Evangeliumstext des Palmsonntags ist ein direkter Hinweis darauf, dass er sich - wie im Titel angekündigt - an die Evangeliumstexte für die Sonn- und Festtage des Kirchenjahres gehalten hat. Tatsächlich war durch Kirchenordnungen in den Hohenloher Herrschaftsgebieten längst geregelt, welcher Bibeltext bei welchem Gottesdienst zu predigen wäre. Dass es darüber aber immer wieder Diskussionen und Klärungsbedarf gab, zeigt z.B. ein Schriftwechsel der Generalsuperintendentur in Öhringen, dem Sitz des gemeinsamen Konsistoriums der Hohenloher Herrschaften. ${ }^{19}$ Dort hatten Pfarrer für freie Textwahl plädiert mit dem interessanten Argument, dass ihnen zum immer wieder gleichen Text nichts Neues mehr einfalle. Mayer war 53 Jahre in Kupferzell! Das Konsistorium hatte für dieses Argument durchaus Verständnis, wollte aber die Gemeinschaft in Sachen Liturgie mit den anderen lutherischen Kirchen nicht aufgeben.

Ob Mayer die im Band vorgelegten Predigten tatsächlich in einem Kalenderjahr nacheinander gehalten oder aus mehreren Jahren zusammengestellt hat, konnte ich nicht herausfinden.

\section{Gesamtwürdigung}

Die vorgestellten Predigten zeigen uns Johann Friedrich Mayer als einen - fast möchte man sagen: wie könnte es auch anders sein - typischen Vertreter seiner Zeit - im Positiven wie im Negativen.

Er war einer von vielen fortschrittsbegeisterten Pfarrern, die sich in agrar-ökonomisch-naturwissenschaftlichen Gesellschaften engagierten. Sie waren in ganz Deutschland verbreitet, oft nur örtliche Größen, aber auch - wie Mayer - über-

17 Ebd., S. 50 f.

18 Ebd., S. 276.

19 HZAN La 25, Bü 683. 
regional bekannt; in Württemberg z.B. Jeremias Höslin in Böhringen bei Urach oder Johann Gottlieb Steeb in Grabenstetten. ${ }^{20}$ Im Elsass wäre zu erwähnen Johann Friedrich Oberlin in Waldersbach im Steintal in den Vogesen. ${ }^{21}$ Sie alle wollten - wie Mayer - begeistert vom fortschreitenden Erkenntnisgewinn in Philosophie und Naturwissenschaft dieses gute Wissen zur Wohlfahrt ihrer Gemeinden weitergeben. Denn darin sahen sie Gottes Geist am Werk, der ja ,in alle Wahrheit führt" (Joh.16, 13).

Selbst begeistert vom fortschreitenden Erkenntnisgewinn in Philosophie und Naturwissenschaft will er dieses gute Wissen zur Wohlfahrt seiner Gemeinde weitergeben. Er sieht darin den Geist Gottes am Werk, der ja in alle Wahrheit führt (Johannes 16, 13).

Die Hlg. Schrift dient zu allem und gibt Unterricht auf alles, damit der Mensch vollkommen werde, zu allem Guten geschickt. Sie lehrt uns aufs Ewige mit Fleiß und hat in sich überall die besten Regeln auf das Irdische in sich verstreut. ${ }^{22}$ Alle Grundregeln des ganzen Glücks für alle Stände, für jeden Beruf auf Zeit und Ewigkeit hört man da; und gesammelt in Schriften liest man sie da. ${ }^{23}$

Mayer versteht die Bibel als eine Einführung in die vernünftige Wahrheit, die wenn sie nicht hier stehen würde - heute von jedem Einsichtigen gefunden werden könnte. Wunder sind für ihn deshalb auch nur jetzt noch nicht vernünftig zu erklärende Ereignisse, die es zu entziffern gilt. Ein leichtes Zögern spürt man bei Mayer immerhin bei der Auferstehung. Die Bibel ist für ihn eher eine Spruchsammlung, die hilfreiches Beweismaterial zur Untermauerung eigener vernünftiger Einsichten gibt.

Für die Predigt folgt daraus: Sie zielt nicht auf die Vergegenwärtigung eines historisch-abständigen, kontingenten Ereignisses. Sie braucht bloß die zeitlos gültige Wahrheit zu wiederholen, die dem Vernünftigen unmittelbar einleuchtet. So hilft die Predigt tatsächlich - wie im Titelprogramm angekündigt - den Bürgern und Bauern zum wahren Glauben im Sinne von mehr vernünftiger Erkenntnis und steigert dadurch deren wahre Lebensweisheit. Ob das aber zu mehr ächter christlicher Religion führt? Begriffe wie Unglaube, Glaubenszweifel, Anfechtung, Gottferne, Sünde, Erlösung kommen bei Mayer fast nicht vor. Unglaube ist bloß Defizit oder Fehltritt im Denken. Biblische Tröstung und Stärkung kann deshalb auch nicht mehr sein als ein Appell, besser zu werden, verständiger, genauer und schärfer zu erkennen. Dass Glaube noch nicht Wissen ist, sondern eine Erkenntnis mit noch dunklen Flecken (1. Korinther 13, 12), ist dieser Theologie fremd.

20 Günther Franz: Pfarrer als Wissenschaftler. In: Martin Greiffenhagen (Hg.): Das evangelische Pfarrhaus. Eine Kultur- und Sozialgeschichte. Stuttgart 1984, S. 277-294.

21 Albert Meier: Oberlin, Johann Friedrich (Jean Frédéric). In: Neue Deutsche Biographie 19. Berlin 1999, S. 395 f; Loïc Chalmel: Oberlin. Ein Pfarrer der Aufklärung. Potsdam 2012; Thomas Weiß: Oberlin, Waldersbach. Eine Begegnung. Tübingen 2016.

22 Predigtsammlung, S. 43 f.

23 Ebd., S. 244. 
Mathias Claudius - der Zeitgenosse Johann Friedrich Mayers - kritisiert einmal, dass Aufklärung zwar klug, aber nicht gut mache. Die Denkkraft ist ja nur die Hälfte des Menschen - und noch dazu die unrechte Hälfte. Also auf eine gewisse Gestalt des inwendigen Menschen kommt es an; auf eine gewisse innerliche Denkart, Haltung, Fassung.

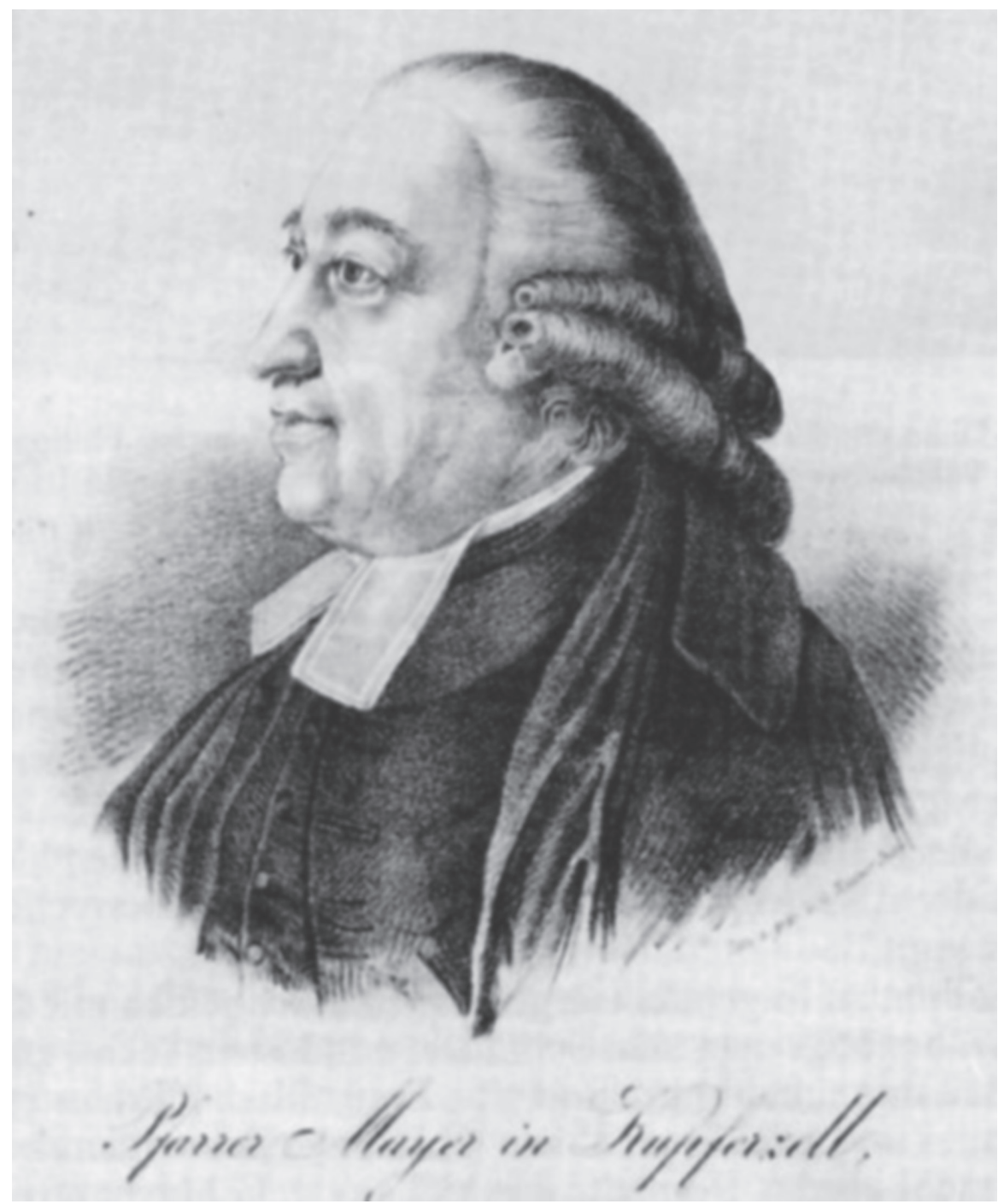

Der Kupferzeller „,Gypsapostel“ Johann Friedrich Mayer, mit Aufschrift Gebhardt.sc. Lithographie von G. M. Eckert. Veröffentlicht auch im ,Wochenblatt für Land- und Forstwirthschaft" 1858. 


\section{Exkurs: Mayers Sicht der Juden}

Wie erwähnt, kommen im ganzen Predigtband Juden nur im Zusammenhang der genannten biblischen Texte vor. Hat es im Hohenloher Fürstentum keine Juden gegeben? In seinem „Lehrbuch für die Land- und Haußwirthe“ äußert sich Mayer - anders als im Predigtband - in durchaus politischem Sinn dezidiert über die Juden und hat seine ganz eigene Sicht. ${ }^{24}$

Juden als sesshafte Bürger gibt es im Fürstentum nicht. Aber als Viehhändler, Geldgeber und Handelspartner sind sie eine gewohnte Realität. Mayer referiert gewissermaßen zuerst die gängigen Vorurteile und Unterstellungen: Der Jud glaubet, daß er vermöge göttlichem Ausspruche Recht und Eingeständnuß die Vorrechte besitze jeden, der nicht Jud ist, ohne alle Sünde und Strafe betriegen, berauben und plündern zu dürfen; darnach handelt er stets, wo er die Menschen nicht scheuet, das Schwerdt nicht sieht und den Galgen umgehen zu können vermeynet. ${ }^{25}$

Dann aber fährt er mit seiner eigenen Einstellung fort - überraschend modern und eben aufgeklärt: mich deucht nur dieses einem Lande sehr nützlich: statt fremde Juden ins Land zu lassen da zu handeln und ihnen die Freiheit zu gönnen, täglich für etwas Zoll mehrere Gulden zu gewinnen und sie in andere Länder zu verschleppen, sollte man ihnen zwar in jedem Lande bei guter christlicher Aufsicht auf ihre ihnen unsündlich scheinende Betrügereien den Handel erlauben; aber sie sollten sich darinnen auch ansetzen, gleiche Zahlungen wie andere Unterthanen übernehmen, gleiche Last tragen, im Frieden und im Krieg dienen oder für ihr Geld ihren Mann aufstellen. Die Dultung, die Frucht der allgemeinen Liebe, heißt mich so schreiben. Und weiter: Man behandelt den Juden vielfältig zu hart. Der Druck druckt ihm seine Seele zu Boden und erstickt dem Geist gänzlich. Hasse ich doch nichts so sehr als den Intoleranten. ${ }^{26}$

24 Johann Friedrich Mayer: Lehrbuch für die Land- und Haußwirthe in der pragmatischen Geschichte der gesamten Lands- und Haußwirthschafft des Hohenlohe Schillingsfürstischen Amtes Kupferzell. Nürnberg 1782, S. 243.

25 Ebd., S. 244.

26 Ebd., S. $247 \mathrm{ff}$. 